\title{
Contribution to the knowledge of the fauna of Ichneumonidae (Hymenoptera: Ichneumonoidea) from Iran
}

\author{
HaSsan GhahaRI ${ }^{1}$ and ReIJO Jussila ${ }^{2}$ \\ ${ }^{1}$ Department of Plant Protection, Yadegar - e- Imam Khomeini (RAH) Branch, Islamic Azad University, Tehran, Iran. - \\ hghahari@yahoo.com \\ 2 Zoological Museum, Section of Biodiversity and Environmental Sciences, Department of Biology, FI-20014 University of Turku, \\ Finland. - reijo.jussila@utu.fi \\ Published on 2016-06-30
}

\section{Summary}

In this paper, 17 species of the family Ichneumonidae (Hymenoptera) from 9 subfamilies, Anomaloninae, Cremastinae, Cryptinae, Ctenopelmatinae, Ichneumoninae, Orthocentrinae, Pimplinae, Tersilochinae and Tryphoninae, are listed from different regions of Iran. Three species, Gelis anthracinus (Förster, 1850), Eusterinx (Dallatorrea) circaea Rossem, 1982 and Barycnemis agilis (Holmgren, 1860), are new records for the fauna of Iran.

\section{Key words}

Hymenoptera, Ichneumonidae, faunistic list, new records, Iran

\section{Zusammenfassung}

In dieser Arbeit werden 17 Arten der Unterfamilien Anomaloninae, Cremastinae, Cryptinae, Ctenopelmatinae, Ichneumoninae, Orthocentrinae, Pimplinae, Tersilochinae und Tryphoninae der Familie Ichneumonidae (Hymenoptera) aus verschiedenen Regionen Irans nachgewiesen. Drei Arten, Gelis anthracinus (FöRster, 1850), Eusterinx (Dallatorrea) circaea Rossem, 1982 und Barycnemis agilis (Holmgren, 1860), sind Erstnachweise für die Fauna Irans.

\section{Introduction}

The Ichneumonidae represents the largest family of Hymenoptera with 48 generally recognized subfamilies, 1579 genera and 24.281 described species (Yu et al. 2012; ÇoruH et al. 2014). The probable number of the species is estimated to be more than 100000 species, and the number of known species increases rapidly in the world (ÇORUH et al. 2014). Members of this family are considered to be the one of the most important groups of parasitoids of various pests in different orders of insects. Hosts are among others Lepidoptera, Coleoptera, Diptera and to a less extent spiders and the egg sacs of spiders and pseudoscorpions (WAHL \& SHARKEY 1993; FINCH
2005; ÇORUH \& Kolarov 2010). Some of the ichneumonids have been used successfully as biocontrol agents and given the largely undocumented fauna there is a huge potential for their use in managed biocontrol programs (Gupta 1991).

Several studies on the fauna of Iranian Ichneumonidae have been conducted by the first author since 2005 by publishing the catalogue of Iranian Ichneumonidae (Kolarov \& Ghahari 2005). This is followed by many other faunistic papers that are published by different authors, examples are those by BARAHOEI et al. (2012) with 502 species belonging to 189 genera and 
24 subfamilies; Ghahari 2014; Ghahari \& Jussila 2014a, b, 2015; GHAHARI et al. 2014a, b; BARAHOEI et al. 2014a, b, 2015, etc. In the present study, a survey covering some regions of Iran was done resulting in new faunistic data as well as three new records to the fauna of Iran.

\section{Material and Methods}

Specimens were collected by Malaise traps and sweeping nets from some regions of Iran. The collected materials were put in alcohol $75 \%$ or mounted on triangular labels and were examined with a stereoscopic binocular microscope. The specimens are deposited in the collections of the authors. The subfamilies, genera and species are listed in alphabetic order according the recent Interactive Catalogue of World Ichneumonidae (Yu et al. 2012). The distributional records were also used based on $\mathrm{Yu}$ et al. (2012).

\section{Results and Discussion}

In total 17 ichneumonid species from 16 genera (Anomalon PANZER, Barycnemis Förster, Barytarbes Förster, Coelichneumon Thomson, Dirophanes FÖRSTER, Eusterinx FöRster, Exeristes Förster, Gelis Thunberg, Itoplectis Förster, Kristotomus MAson, Pimpla Fabricius, Scambus Hartig, Temelucha Förster, Theronia Holmgren, Tryphon Fallén, and Virgichneumon HeInRICH) were collected and identified from some regions of Iran. This work contributes to add some new records to the Iranian fauna; however, more investigations and complementary sampling are needed to get a good knowledge of the distribution and trophic associations of ichneumonid wasps in Iran. The list of species with distribution data is given below.

\section{Subfamily Anomaloninae ViereCK, 1918}

\section{Genus Anomalon PANZER, 1804}

\section{Anomalon cruentatum (GEOFFROY, 1785)}

Material examined: Fars province, Kazerun, $29^{\circ} 35^{\prime} \mathrm{N}$ $51^{\circ} 40^{\prime} \mathrm{E}, 1$ ㅇ , April 2009. Kerman province, Jiroft, $28^{\circ} 50^{\prime} \mathrm{N}$ $57^{\circ} 35^{\prime} \mathrm{E}, 1$ ㅇ, summer 2010 .

Previous records from Iran: Ardabil (Masnadi \& Jussila 2009), East Azarbaijan (Ghahari \& Jussila 2011a), Isfahan (Barahoei et al. 2015), Khorasan (Barahoei et al. 2014a), Sistan \& Baluchistan (Barahoei et al. 2012), Yazd (Zarepour et al. 2009 as A.foliator), Iran (no locality cited) (Kolarov \& Ghahari 2005).

General distribution: Afghanistan, Austria, Azerbaijan, Belarus, Belgium, Bulgaria, Canary Islands, China, Croatia, Cyprus, Czech Republic, Egypt, Finland, France,
Germany, Greece, Hungary, India, Iran, Israel, Italy, Japan, Jordan, Kazakhstan, Korea, Latvia, Lebanon, Libya, Lithuania, Malta, Moldova, Mongolia, Myanmar, Netherlands, Pakistan, Poland, Portugal, Romania, Russia, Spain, Sri Lanka, Sweden, Switzerland, Tunisia, Turkey, Turkmenistan, Ukraine, UK, Uzbekistan, former Yugoslavia.

\section{Subfamily Cremastinae FöRSTER, 1869}

\section{Genus Temelucha FörSTER, 1869}

Temelucha arenosa (SzÉPLIGETI, 1899)

Material examined: Hamadan province, Malayer, $34^{\circ} 20^{\prime} \mathrm{N} 48^{\circ} 45^{\prime} \mathrm{E}, 1$ 웅, October 2014.

Previous records from Iran: Mazandaran (Ghahari \& Jussila 2011a), Tehran (Ghahari \& Jussila 2010a).

General distribution: Bulgaria, Czech Republic, France, Germany, Hungary, Iran, Ireland, Lithuania, Macedonia, Netherlands, Poland, Romania, Spain, Sweden, Turkey, UK, former Yugoslavia.

\section{Subfamily Cryptinae KIRBY, 1837}

\section{Genus Gelis ThunBeRg, 1827}

Gelis anthracinus (FöRSTER, 1850)

Material examined: Ardabil province, Germi, $39^{\circ} 00^{\prime} \mathrm{N}$ $47^{\circ} 57^{\prime} \mathrm{E}, 1$ ㅇ, September 2011. New record for Iran.

General distribution: Austria, Bulgaria, Czech Republic, Finland, France, Germany, Greece, Hungary, Ireland, Italy, Latvia, Norway, Poland, Romania, Russia, Spain, Sweden, Ukraine, UK.

Subfamily Ctenopelmatinae FörsTER, 1869

Genus Barytarbes FöRSTER, 1869

\section{Barytarbes superbus ScHMIEDEKNECHT, 1914}

Material examined: Zanjan province, Abhar, $36^{\circ} 16^{\prime} \mathrm{N}$ $49^{\circ} 03^{\prime} \mathrm{E}, 1$ ㅇ, 1 ơ , June 2011.

Previous records from Iran: Golestan (Ghahari \& Jussila 2011a).

General distribution: Algeria, Bulgaria, Czech Republic, France, Germany, Greece, Hungary, Iran, Israel, Lithuania, Romania, Spain, Tunisia, Turkey, former Yugoslavia. 
Subfamily Ichneumoninae LATREILLE, 1802

Genus Coelichneumon Thomson, 1893

\section{Coelichneumon biannulatus (GRAVENHORST, 1820)}

Material examined: Zanjan province, Abhar, $36^{\circ} 16^{\prime} \mathrm{N}$ $49^{\circ} 03^{\prime} \mathrm{E}, 1$ \% , June 2011.

Previous records from Iran: Ardabil (Kolarov \& Ghahari 2008), Mazandaran (Ghahari \& Jussila 2010b).

General distribution: Austria, Azerbaijan, Belarus, Belgium, Bulgaria, Czech Republic, Finland, France, Germany, Iran, Netherlands, Norway, Poland, Russia, Sweden, Switzerland, Turkey, Ukraine, UK.

\section{Genus Dirophanes FöRSTER, 1869}

Dirophanes invisor (THUNBERG, 1824)

Material examined: Ardabil province, Germi, $39^{\circ} 00^{\prime} \mathrm{N}$ $47^{\circ} 57^{\prime} \mathrm{E}, 2$ 오 , September 2011.

Previous records from Iran: Guilan (Kolarov \& Ghahari 2008), West Azarbaijan (Ghahari \& Jussila 2011c).

General distribution: Algeria, Andorra, Austria, Azerbaijan, Belarus, Belgium, Bulgaria, Croatia, Czech Republic, Denmark, Estonia, Finland, France, Germany, Hungary, Iran, Ireland, Italy, Latvia, Macedonia, Moldova, Netherlands, Norway, Poland, Romania, Russia, Slovakia, Spain, Sweden, Switzerland, Turkey, Ukraine, UK, former Yugoslavia.

\section{Genus Virgichneumon HeINRICH, 1977}

\section{Virgichneumon callicerus (GravenHORST, 1820)}

Material examined: Khuzestan province, Izeh, $31^{\circ} 54^{\prime} \mathrm{N}$ 495'ㄹ, 1 ㅇ, April 2010.

Previous records from Iran: Ardabil (Masnadi \& Jussila 2008), East Azarbaijan (Kolarov \& Ghahari 2008).

General distribution: Austria, Azerbaijan, Belarus, Belgium, Bosnia-Hercegovina, Bulgaria, China, Croatia, Czech Republic, Finland, France, Germany, Hungary, Iran, Italy, Kazakhstan, Moldova, Netherlands, Norway, Poland, Romania, Russia, Slovakia, Spain, Sweden, Switzerland, Turkey, Ukraine, UK.
Subfamily Orthocentrinae FöRSTER, 1869

Genus Eusterinx FörsTER, 1869

Eusterinx (Dallatorrea) circaea Rossem, 1982

Material examined: Golestan province, Gorgan, $36^{\circ} 50^{\prime} \mathrm{N}$ $54^{\circ} 30^{\prime} \mathrm{E}, 2$ ․ July 2013. New record for Iran.

General distribution: Austria, Finland, Germany, Italy, Kazakhstan.

\section{Subfamily Pimplinae WeSMAeL, 1845}

\section{Genus Exeristes FönSTER, 1869}

\section{Exeristes arundinis (KRIECHBAUMER, 1887)}

Material examined: Golestan province, Gorgan, $36^{\circ} 50^{\prime} \mathrm{N}$ $54^{\circ} 30^{\prime} \mathrm{E}, 1$ ㅇ , July 2013.

Previous records from Iran: Golestan (Ghahari \& Jussila 2011b), Guilan (Ghahari \& Jussila 2012), Mazandaran (Ghahari \& Jussila 2011a; Ghahari \& Schwarz 2011), Zanjan (Kolarov \& Ghahari 2006).

General distribution: Austria, Belarus, Bulgaria, China, Czech Republic, Finland, France, Germany, Hungary, Iran, Kazakhstan, Latvia, Macedonia, Mongolia, Poland, Romania, Russia, Spain, Switzerland, Turkey, Turkmenistan, Ukraine, former Yugoslavia.

\section{Genus /toplectis FöRSTER, 1869}

$$
\text { Itoplectis maculator (FABRICIUS, 1775) }
$$

Material examined: Khuzestan province, Izeh, $31^{\circ} 54^{\prime} \mathrm{N}$ 49 $59^{\prime} \mathrm{E}, 1$ ㅇ , April 2010.

Previous records from Iran: Lorestan (Ghahari \& Gadallah 2015), Tehran (Mohammadi et al. 2013), West Azerbaijan (Kolarov \& Ghahari 2006).

General distribution: Albania, Armenia, Austria, Azerbaijan, Belarus, Belgium, Bulgaria, Canary Islands, Croatia, Czech Republic, Denmark, Estonia, Finland, France, Georgia, Germany, Greece, Hungary, Iran, Ireland, Italy Latvia, Lithuania, Luxembourg, Macedonia, Malta, Moldova, Mongolia, Morocco, Netherlands, Norway, Poland, Portugal, Romania, Russia, Serbia \& Montenegro, Slovakia, Spain, Sweden, Switzerland, Tunisia, Turkey, Ukraine, UK, former Yugoslavia. 


\section{Genus Pimpla FABRICIUS, 1804}

\section{Pimpla contemplator (MüLLER, 1776)}

Material examined: Northern Khorasan province,

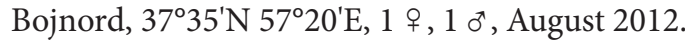

Previous records from Iran: Golestan (Kolarov \& Ghahari 2006), Sistan \& Baluchestan (Barahoei et al. 2013a).

General distribution: Albania, Armenia, Austria, Azerbaijan, Belarus, Belgium, Bulgaria, Canary Islands, Czech Republic, Denmark, Egypt, Finland, France, Georgia, Germany, Hungary, Iran, Ireland, Italy, Japan, Latvia, Lithuania, Luxembourg, Macedonia, Moldova, Mongolia, Netherlands, Norway, Poland, Romania, Russia, Serbia \& Montenegro, Spain, Sweden, Switzerland, Turkey, Turkmenistan, Ukraine, UK, former Yugoslavia.

\section{Genus Scambus HARTIG, 1838}

\section{Scambus brevicornis (GRAVENHORST, 1829)}

Material examined: Ardabil province, Germi, $39^{\circ} 00^{\prime} \mathrm{N}$ 475ㅡ'E, 2 우, September 2011.

Previous records from Iran: Kordestan (Kolarov \& Ghahari 2006).

General distribution: Austria, Azerbaijan, Belgium, Bulgaria, Canada, China, Czech Republic, Denmark, Finland, France, Georgia, Germany, Greenland, Hungary, Iceland, Iran, Ireland, Italy, Japan, Kazakhstan, Latvia, Lithuania, Luxembourg, Macedonia, Moldova, Mongolia, Netherlands, Norway, Poland, Romania, Russia, Serbia \& Montenegro, Slovakia, Spain, Sweden, Switzerland, Tunisia, Turkey, USA, Ukraine, UK, former Yugoslavia.

\section{Genus Theronia HOLmgren, 1859}

\section{Theronia atalantae (PoDA, 1761)}

Material examined: Northern Khorasan province,

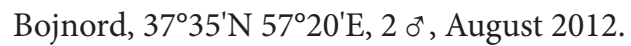

Previous records from Iran: Chaharmahal \& Bakhtiari (Kolarov \& Ghahari 2006).

General distribution: Austria, Azerbaijan, Belarus, Belgium, Bulgaria, Canada, China, Croatia, Czech Republic, Denmark, Finland, France, Germany, Greece, Hungary, India, Iran, Italy, Japan, Korea, Latvia, Macedonia, Moldova, Netherlands, Poland, Romania, Russia, Serbia \& Montenegro, Slovakia, Slovenia, Spain, Sweden, Switzerland, Turkey, Turkmenistan, USA, Ukraine, UK, former Yugoslavia.

\section{Subfamily Tersilochinae SCHMIEDEKNECHT, 1910}

\section{Genus Barycnemis FöRSTER, 1869}

\section{Barycnemis agilis (HoLmgren, 1860)}

Material examined: East Azarbaijan province, Maragheh, $37^{\circ} 23^{\prime} \mathrm{N} 46^{\circ} 24^{\prime} \mathrm{E}, 1$ 우 , September 2011. New record for Iran.

General distribution: Belarus, Bulgaria, Canada, Czech Republic, Denmark, Finland, Germany, Kyrgyzstan, Lithuania, Mongolia, Norway, Poland, Russia, Sweden, USA, Ukraine.

\section{Subfamily Tryphoninae SHUCKARD, 1840}

Genus Kristotomus Mason, 1962

Kristotomus laetus (GravenHORST, 1829)

Material examined: Ardabil province, Germi, $39^{\circ} 00^{\prime} \mathrm{N}$ 47ํ5'ㄹ, 1 우, September 2011.

Previous records from Iran: Sistan \& Baluchestan (Kolarov \& Ghahari 2006).

General distribution: Armenia, Austria, Belgium, Bulgaria, Czech Republic, Denmark, Finland, France, Georgia, Germany, Greece, Hungary, Iran, Ireland, Italy, Latvia, Lithuania, Mongolia, Netherlands, Norway, Poland, Romania, Russia, Slovakia, Spain, Sweden, Switzerland, Turkey, Ukraine, UK.

\section{Genus Tryphon FalLÉN, 1813}

Tryphon rutilator (LINNAEUS, 1761)

Material examined: Mazandaran province, Neka, $36^{\circ} 30^{\prime} \mathrm{N} 53^{\circ} 32^{\prime} \mathrm{E}, 20^{*}$, summer 2009. Northern Khorasan province, Bojnord, $37^{\circ} 35^{\prime} \mathrm{N} 57^{\circ} 20^{\prime} \mathrm{E}, 1$ 우 $10^{\star}$, August 2012.

Previous records from Iran: Golestan (Kolarov \& Ghahari 2005).

General distribution: Albania, Armenia, Austria, Azerbaijan, Belarus, Belgium, Bulgaria, Croatia, Czech Republic, Denmark, Estonia, Finland, France, Georgia, Germany, Greece, Hungary, Iran, Italy, Latvia, Lithuania, Luxembourg, Moldova, Netherlands, Norway, Poland, Romania, Russia, Spain, Sweden, Switzerland, Syria, Turkey, Ukraine, UK, former Yugoslavia. 


\section{Tryphon thomsoni ROMAN, 1939}

Material examined: Khuzestan province, Izeh, $31^{\circ} 54^{\prime} \mathrm{N}$ 4959'E, 1 ㅇ, April 2010.

Previous records from Iran: Golestan (Ghahari \& Jussila 2012), Guilan (Kolarov \& Ghahari 2006), Markazi (Ghahari et al. 2014b).

General distribution: Albania, Armenia, Austria, Azerbaijan, Belarus, Belgium, Bulgaria, Czech Republic, Finland, France, Georgia, Germany, Iran, Ireland, Israel, Italy, Lithuania, Netherlands, Norway, Poland, Russia, Spain, Tajikistan, Turkey, Ukraine, UK.

\section{Acknowledgements}

We wish to express our sincere gratitude to N.S. Gadallah (Cairo University, Egypt) for her valuable role in progress of the project, E. Ruiz-Cancino (Universidad Autónoma de Tamaulipas, Mexico) for editing the manuscript and H. Sakenin (Qaemshahr Islamic Azad University) for loaning some specimens. The research was supported by Islamic Azad University (Yadegar - e- Imam Khomeini (RAH) Branch) and University of Turku.

\section{References}

Barahoei, H.; Rakhshani, E. \& Riedel, M. 2012 : A checklist of Ichneumonidae (Hymenoptera: Ichneumonoidea) from Iran. - Iranian Journal of Animal Biosystematics (IJAB) 8 (2): 83-132.

Barahoei, H.; Rakhshani, E.; Kasparyan, D. R.; Schwarz, M. \& Riedel, M. 2013a: Contribution on the knowledge of Ichneumonidae (Hymenoptera) in the northern part of Sistan and Baluchestan province, Iran. - Acta Zoologica Bulgarica 65 (1): 131-135.

Barahoei, H.; Rakhshani, E.; Fathabadi, K. \& Moradpour, H. 2014a: A survey on the fauna of Ichneumonidae (Hymenoptera) of Khorasane-Razavi province. - Iranian Journal of Animal Biosystematics (IJAB) 10 (2): 145-160.

Barahoei, H.; NAder E. \& Rakhshani, E. 2014b: Cryptinae (Hymenoptera: Ichneumonidae) of Isfahan province, central Iran. - Turkish Journal of Zoology 39: 279-284.

Barahoei, H.; Nader, E. \& Rakhshani, E. 2015: A survey on Ichneumonidae of Isfahan province, central Iran. - Journal of Crop Protection 4(2): $157-166$

Çoruh, S. \& Kolarov, J. 2010: A review of the Turkish Orthopelmatinae (Insecta: Hymenoptera: Ichneumonidae). - Scientific Research and Essays 5 (22): 3518-3521.
Çoruh, S.; Kolarov, J. \& Çoruh, I. 2014: Probles microcephalus (GravenHORST, 1829) a new record for the Turkish fauna (Hymenoptera: Ichneumonidae: Tersilochinae). - Munis Entomology \& Zoology 9 (1): 451-456.

FInCH, O. D. 2005: The parasitoid complex and parasitoid-induced mortality of spiders (Araneae) in a central European woodland. - Journal of Natural History 39 (25): 2339-2354.

GHAHARI, H. 2014: A study on the subfamily Ichneumoninae (Hymenoptera: Ichneumonidae) from Varamin and vicinity, Iran. - Calodema 295: $1-2$.

Ghahari, H. \& Jussila, R. 2010a: A contribution to the knowledge of ichneumon wasps (Hymenoptera: Ichneumonidae) from Iranian cotton fields and surrounding grasslands. - Zoosystematica Rossica 19 (2): $357-360$.

Ghahari, H. \& Jussila, R. 2010b: Some new records of Iranian Ichneumoninae (Hymenoptera: Ichneumonidae). - Linzer biologische Beiträge 42 (2): 1373-1377.

Ghahari, H. \& Jussila, R. 2011a: A contribution to the knowledge of Ichneumonidae (Hymenoptera) from Arasbaran and vicinity, Iran. - Calodema 166: 1-5.

Ghahari, H. \& Jussila, R. 2011b: A study on the ichneumonid wasps (Hymenoptera: Ichneumonidae) from some regions of Iran. - Linzer biologische Beiträge 43 (1): 753-758.

Ghahari, H. \& Jussila, R. 2011c: Five species of Ichneumonidae (Hymenoptera) new to Iran. Zoology in the Middle East 52: 123-124.

Ghahari, H. \& Jussila, R. 2012: A contribution to the knowledge of the ichneumon wasps (Hymenoptera: Ichneumonidae) from Guilan Province, Northern Iran. - Phegea 40 (1b): 1-4.

Ghahari, H. \& Jussila, R. 2014a: A faunistic study on the Ichneumonidae (Hymenoptera: Ichneumonoidea) from the west of Iran. - Linzer biologische Beiträge 46 (2): 1373-1377.

Ghahari, H. \& Jussila, R. 2014b: A study on the subfamily Ichneumoninae (Hymenoptera: Ichneumonidae) from Khorasan province, Iran. - Linzer biologische Beiträge 46 (2): 1367-1371.

Ghahari, H.; Ostovan, H.; Jussila, R. \& Behnood, S. 2014a: A study on Ichneumonidae (Hymenoptera: Ichneumonoidea) from some regions of Khorasan province, north-eastern Iran. - Calodema 296: 1-2.

Ghahari, H.; Jussila, R. \& Gadallah, N. S. 2014b: The species of Ichneumonidae (Hymenoptera: Ichneumonoidea) from Markazi province, Iran. - Wuyi Science Journal 30: 83-91.

Ghahari, H. \& Gadallah, N. S. 2015: A study on the ichneumonid wasps (Hymenoptera: Ichneumonidae) from the province of Lorestan, Iran. - Arquivos Entomóloxicos 13: 329-338. 
Ghahari, H. \& Jussila, R. 2015: Faunistic notes on the Ichneumonid wasps (Hymenoptera: Ichneumonidae) in alfalfa fields in some regions of Iran. - Entomofauna 36 (12): 185-192.

GHAHARI, H. \& Schwarz, M. 2011: A contribution to the knowledge of ichneumonid wasps from Mazandaran province, northern Iran (Hymenoptera, Ichneumonidae). - Spixiana 34 (2): 195-198.

Gupta V. K. 1991: A review of the Exenterine genus Eridolius (Hymenoptera: Ichneumonidae) and description of new species from the Oriental Region. - Oriental Insects 25: 435-446.

Kolarov, J. \& Ghahari, H. 2005: A Catalogue of Ichneumonidae (Hymenoptera) from Iran. - Linzer biologische Beiträge 37: 503-532.

Kolarov, J. \& GHahari, H. 2006: A study of the Iranian Ichneumonidae (Hymenoptera): I. Pimplinae and Tryphoninae. - Zoology in the Middle East 38: 63-68.

Kolarov, J. \& Ghahari, H. 2008: A study of the Iranian Ichneumonidae (Hymenoptera) III. Ichneumoninae. - Acta Entomologica Serbica 13 (1/2): 61-76.

Masnadi, A. \& Jussila, R. 2008: Contribution to the knowledge of ichneumonid wasps of Iran. Subfamilies Ichneumoninae, Pimplinae and Diplazontinae (Hymenoptera, Ichneumonidae). - Entomofauna 29 (22): 293-320.
Masnadi, A. \& Jussila, R. 2009: A contribution to ichneumonid wasps of Iran (Hymenoptera: Ichneumonidae): Anomaloninae, Cremastinae, Ctenopelmatinae, Mesochorinae, Metopiinae and Orthopelmatinae). - Applied Entomology and Phytopathology 76 (2): 11-28.

Mohammadi, A.; Talebi, A. A. \& Zwakhals, K. 2013: A study of the subfamily Pimplinae (Hymenoptera: Ichneumonidae) in the north of Iran, with eleven new species records. - Entomofauna 34 (2): 29-56.

Wahl, D. B. \& Sharkey, M. J. 1993: Superfamily Ichneumonoidea, pp. 358-509. - In: Goulet, H. \& Huber, J. T. (eds), Hymenoptera of the world: An identification guide to families. - Agriculture Canada, Ottawa, vii + 668 pp.

Yu, D. S.; van Achterberg, K. \& Horstmann, K. 2012: World Ichneumonoidea 2011. Taxonomy, Biology, Morphology and Distribution. - Taxapad.com. Canada.

Zarepour, A. R.; TAlebi, A. A. \& Vafaei Shoushtari, R. 2009: Three new species records of Ichneumonid wasps (Hymenoptera, Ichneumonidae) from Yazd, Iran. - Journal of Entomological Research 1 (1): 67-77. 